\title{
Testing the limits of optimizing dual-task performance in younger and older adults
}

\author{
Tilo Strobach ${ }^{1}{ }^{*}$, Peter Frensch ${ }^{2}$, Herrmann Josef Müller ${ }^{1}$ and Torsten Schubert ${ }^{1,2}$ \\ 1 Department Psychology, Ludwig-Maximilians University Munich, Munich, Germany \\ ${ }^{2}$ Department of Psychology, Humboldt-University Berlin, Berlin, Germany
}

\author{
Edited by: \\ Julia Karbach, Saarland University, \\ Germany \\ Reviewed by: \\ Arthur F. Kramer, University of Illinois \\ at Urbana-Champaign, USA \\ Louis Bherer, University of Quebec at \\ Montreal, Canada \\ *Correspondence: \\ Tilo Strobach, Department \\ Psychology, Ludwig-Maximilians- \\ Universität Munich, Leopoldstr. 13, \\ D-80802 Munich, Germany. \\ e-mail: tilo.strobach@psy.Imu.de
}

Impaired dual-task performance in younger and older adults can be improved with practice. Optimal conditions even allow for a (near) elimination of this impairment in younger adults. However, it is unknown whether such (near) elimination is the limit of performance improvements in older adults. The present study tests this limit in older adults under conditions of (a) a high amount of dual-task training and (b) training with simplified component tasks in dual-task situations. The data showed that a high amount of dual-task training in older adults provided no evidence for an improvement of dual-task performance to the optimal dual-task performance level achieved by younger adults. However, training with simplified component tasks in dual-task situations exclusively in older adults provided a similar level of optimal dual-task performance in both age groups. Therefore through applying a testing the limits approach, we demonstrated that older adults improved dual-task performance to the same level as younger adults at the end of training under very specific conditions.

Keywords: cognitive aging, dual-task performance, testing the limits, practice

\section{INTRODUCTION}

In recent years, a number of studies have examined the effect of practice on dual-task performance across different age groups in order to better understand the basic cognitive mechanisms underlying dual-task performance and cognitive aging (Maquestiaux et al., 2004, 2010; Bherer et al., 2005, 2006, 2008; Göthe et al., 2007; Allen et al., 2009; Hartley et al., 2011). In younger adults, some studies have even demonstrated perfect time sharing of two tasks after practice defined by zero performance costs in dual compared to single-tasks (i.e., dual-task costs; Van Selst et al., 1999; Ruthruff et al., 2001, 2003; Schumacher et al., 2001). However, such findings of perfect or near perfect time sharing are lacking in the aging literature on older adults. The aim of the present study was to close this gap. Therefore, we investigated the limits of dual-task performance optimization in older adults (i.e., near perfect or perfect time sharing) and, furthermore, tested the conditions of such optimization.

Investigations of (near) perfect time sharing with practice are interesting for aging research because they may provide more conclusive evidence about cognitive plasticity, its range, and developmental mechanisms in older adults (Bherer et al., 2006). In particular, testing the limits of optimizing dual-task performance in older adults should demonstrate, in the case of complex task situations, the maximum cognitive performance potential or the "latent" reserve capacity of older adults in a more appropriate way than investigating cognitive abilities of older people without extensive practice. Baltes, Lindenberger and colleagues (e.g., Lindenberger et al., 1992; Lindenberger and Baltes, 1995) have argued that the testing the limits approach can lead to an identification of true age-related cognitive decline, rather than overestimate age-related differences due to non-optimized testing conditions, assuming that age-related differences in reserve capacity are more accurately assessed near the limits of performance. Rephrased in the testing the limits terminology, we test the developmental reserve when assessing the limits of optimized dual-task performance in older adults. This test is essential because older adults' difficulty in performing concurrent tasks is one of the most well documented executive control deficits in cognitive aging literature (e.g., Allen et al., 1998; Hartley and Little, 1999; Glass et al., 2000; McDowd and Shaw, 2000; Hartley, 2001; Verhaeghen et al., 2003; Hein and Schubert, 2004; Verhaeghen, 2011). This deficit may result from age-impaired attentional control processes that are related to the substantial modifications observed in the frontal and prefrontal areas of the cerebral cortex during aging (e.g., West, 1996; Raz, 2000).

\section{OPTIMIZING DUAL-TASK PERFORMANCE IN YOUNGER AND OLDER ADULTS}

Meyer and Kieras (1999) outlined conditions for optimal dual-task performance. In particular, the authors listed five prerequisites, which should be fulfilled in order to achieve such performance: "(Condition 1) participants are encouraged to give the tasks equal priority; (Condition 2) participants are expected to perform each task quickly; (Condition 3) there are no constraints on temporal relations or serial order among responses; (Condition 4) different tasks use different perceptual and motor processors; and (Condition 5) participants receive enough practice to compile complete production rule sets for performing each task" (p. 54).

Previous attempts to compare practice-related improvements in older and younger adults' dual-task processing have provided impressive findings concerning cognitive plasticity in old age; however, unfortunately, they have not yet considered all of the conditions mentioned above and consequently their findings may not be fully conclusive regarding the limits of practice-related changes 
in older adults' dual-task performance. For instance, Maquestiaux et al. (2010) applied a dual-task practice situation that emphasizes response speed for and extensive practice of only one component task (i.e., Conditions 1, 3, and 5 of Meyer and Kieras, 1999, were not implemented). In a different line of research, Bherer et al. $(2005,2006)$ did not include all conditions when applying similar perceptual and motor processors on the component tasks (i.e., Condition 4 was not implemented).

One such situation including conditions for optimal dual-task performance was applied in younger and older adults by Strobach et al. (2012b), see also Schumacher et al., 2001). The authors asked participants to perform a training paradigm that consisted of tasks with different perception and motor components (Condition 4): a visual-manual (i.e., the visual task) and an auditory-vocal choice RT task (i.e., the auditory task). During training with these tasks (Condition 5), three different trial types were performed: participants performed only one of the two tasks in single-task blocks (single-task trials); in mixed blocks, participants either responded to only one task (i.e., mixed single-task trials) or actually executed two motor responses to simultaneously presented stimuli in two different tasks (dual-task trials with stimulus onset asynchrony, $\mathrm{SOA}$, of $0 \mathrm{~ms}$ ). Participants were instructed to respond as quickly and as accurately as possible with equal priority and with no prespecified serial order to both stimuli in these trials (Conditions 1,2, and 3). They received adaptive and continuous on-screen feedback as well as performance-based monetary bonuses for optimized RT and error performance.

The training RTs in single-task, mixed single-tasks, and dualtask trials up to Session 8 (younger adults) and Session 12 (older adults) are summarized in Figure 1 and Tables 1 and 2. Dualtask costs are illustrated by the mean difference of dual-task and mixed single-task trials. At the end of eight training sessions, these dual-task costs were extremely reduced in younger adults (see also Schumacher et al., 2001; Hazeltine et al., 2002; Tombu and Jolicoeur, 2004; Strobach et al., 2008; Liepelt et al., 2011; Strobach et al., in press; for an adaption to a task switching situation see Strobach et al., 2012a). These findings demonstrate that in dual-task situations, implementing the prerequisite conditions for optimal dual-task performance (Meyer and Kieras, 1999), younger adults show nearly eliminated dual-task costs, i.e., near perfect time sharing, at the end of training; for the reasons for the not perfect but near perfect time sharing in this design see "General Discussion.” In older adults, however, dual-task costs were still relatively high after the same amount of training (i.e., eight sessions) and even after four additional training sessions when compared to the reduced costs in younger adults. Thus, these data provide no evidence for near perfect time sharing in older adults when testing the limits of cognitive functioning in this age group (e.g., Baltes and Kliegl, 1992).

However, it is conceivable that the defined conditions of Meyer and Kieras (1999) enable near perfect time sharing in younger and older adults. However, these conditions were not appropriately set to the requirements of older adults' cognitive processing and learning functions in Strobach et al. (2012b). Consequently, the aim of the present paper is to modulate task and training characteristics in the dual-tasks of Strobach et al. to create conditions for near perfect time sharing in older adults. In fact, we

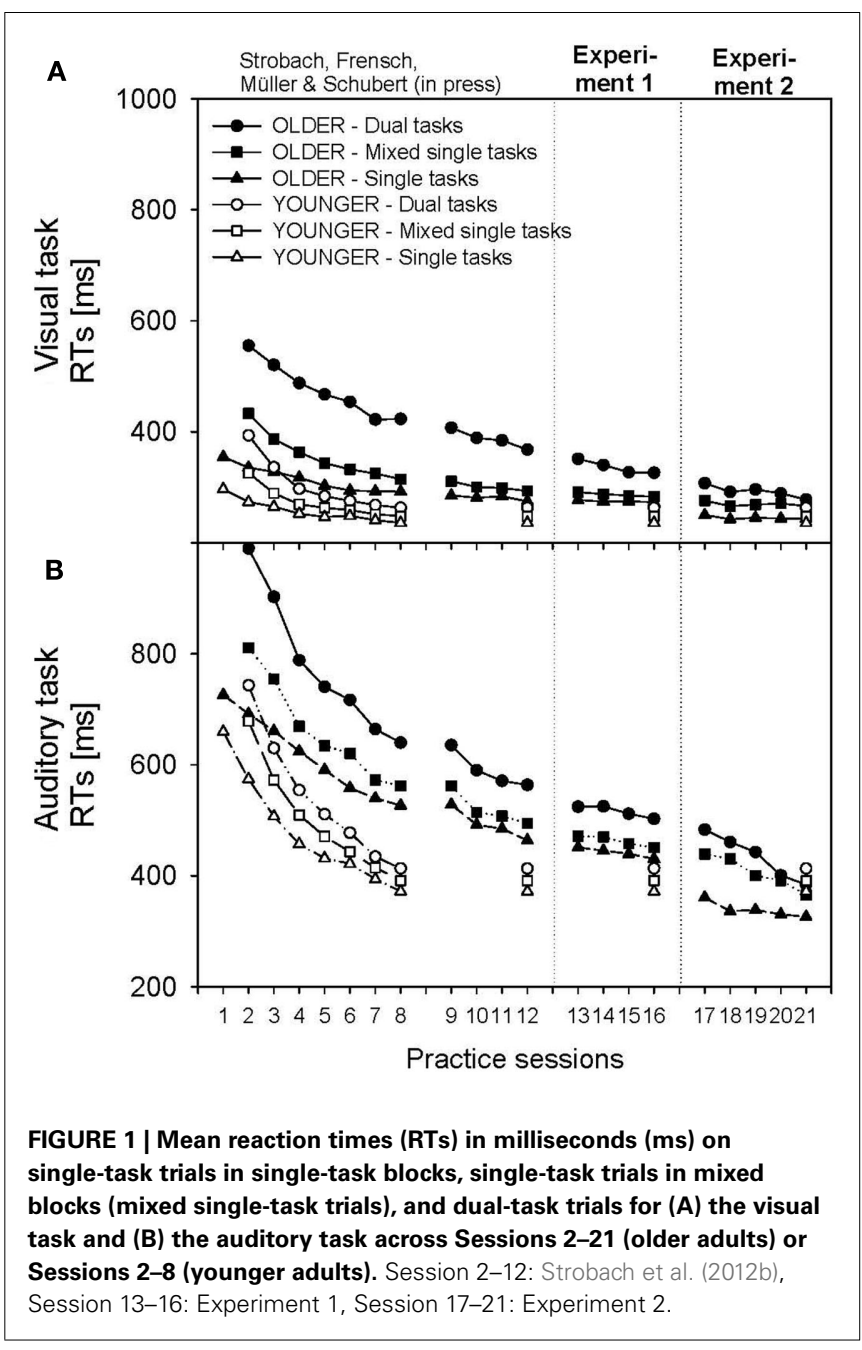

modulated two crucial factors of this dual-task training procedure: (1) we increased the amount of training (Experiment 1) and (2) we simplified the included component tasks (Experiment 2).

\section{EXPERIMENT 1}

One potential explanation for the difference in the dual-task costs between younger and older adults at the end of practice could be the different initial costs in both age groups (e.g., also see Allen et al., 2009). The reduction of these higher costs in older adults to the level of reduced costs in younger adults may require an increased amount of practice in the former group. For instance, this requirement may result from the older adults' slower speed in automatizing task sets during practice (e.g., Kramer et al., 1995). Thus, one possible method to optimize the level of dual-task performance in older adults is to increase the amount of training in this group of participants. This increase in the amount of training is similar to a strategy applied by Maquestiaux et al. (2008) in younger adults. Participants in that study conducted an increased amount of task training compared to a previous study by Ruthruff et al. (2006) to provide optimal conditions for eliminating dualtask performance costs. After this increase, a larger proportion of younger adults performed dual-tasks at an optimized level. 
Table 1 | Error rates in percent in single-task trials of single-task blocks, single-task trials in mixed blocks (mixed single-task trials), and dual-task trials for the visual task in older and younger adults across Sessions 2-12 (older adults) or Sessions 2-8 (younger adults).

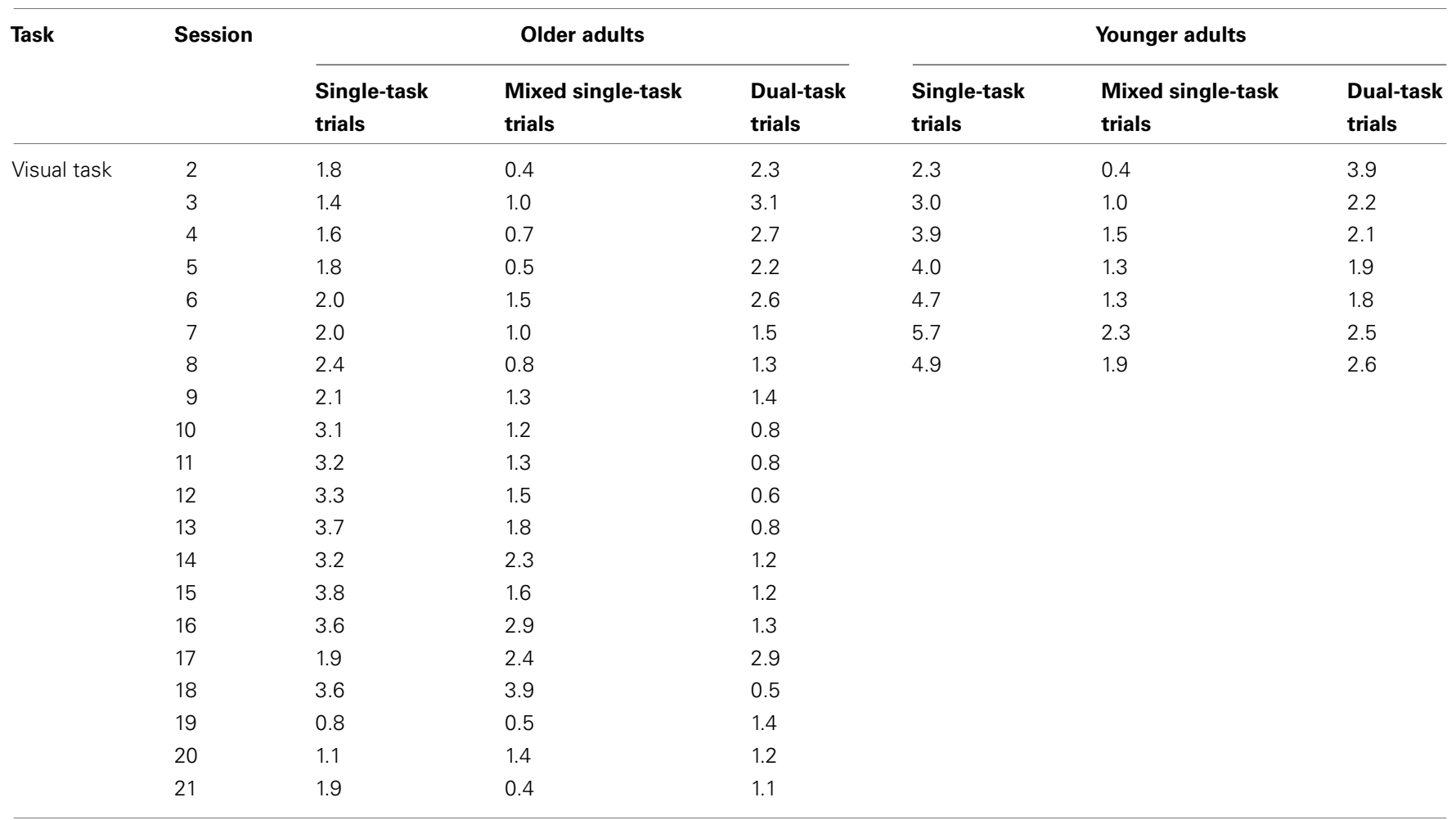

Session 2-12: Strobach et al. (2012b), Session 13-16: Experiment 1, Session 17-21.

Findings also exist about the effects of an increased training amount on dual-task performance through our study with older adults (Strobach et al., 2012b) demonstrating that such increase leads to improved dual-task performance even with prior training, i.e., after four additional sessions following eight prior training sessions.

In fact, in Experiment 1, we increased the amount of dual-task training by adding four sessions immediately after the end of the 12 sessions with the identical older adults of Strobach et al. (2012b). Such a prolongation of training with an identical group of participants makes a novel contribution of the present experiment by testing whether prolonged practice after 12 training sessions enabled older adults to arrive at a performance limit (i.e., near perfect time sharing). This prolongation is plausible because dualtask performance improved until Session 12 and the limit was not attained in this session. Note that we refer to the additional sessions in the following sections of Experiment 1 as Session 13-16 as these sessions immediately followed the Sessions 1-12 reported in Strobach et al. The performance after combined training of 16 sessions with these older adults were compared with the optimized dual-task "target" performance, i.e., reduced dual-task costs reflecting near perfect time sharing, in younger adults after the eighth training session. In this way, we doubled the amount of training in older compared to younger adults before we assessed dual-task performance. If this doubling produces conditions for near perfect time sharing in older adults, we would expect the same level of dual-task performance, i.e., (extremely) reduced dual-task costs, at the end of training in younger adults (i.e., Session 8) and older adults (i.e., Session 16). However, if such doubling is not sufficient to produce conditions for near perfect time sharing in older adults, dual-task costs should not be (extremely) reduced in this age group and should increase the amount of younger adults' costs.

\section{METHOD}

Participants

Ten older adults (mean age $=63.3$ years, $\mathrm{SD}=3.4$, range $57-$ 68, 5 female) were recruited from university courses for senior adults at LMU Munich. Alternatively, the 10 younger adults (mean age $=22.7$ years, $\mathrm{SD}=3.3$, range 19-29, 5 female) were recruited from the university's bachelor and diploma courses. Older and younger adults were paid eight Euros per session plus performance-based monetary bonuses for their participation (for bonus details see Procedure and Design). All participants were generally well educated, with older adults reporting more years of education ( $M=18.0$ years, $\mathrm{SD}=3.9$ years $)$ than younger adults $(M=14.2$ years, $\mathrm{SD}=1.4$ years $), t(18)=2.962, p<0.01$; this higher number years of education in older adults may follow from the prerequisite condition of university courses for senior adults of a prior, completed study. On a five-point health rating scale $(1=$ poor health; $5=$ excellent health), older and younger adults gave similar mean self-ratings of $4.4(\mathrm{SD}=0.7)$ and 3.7 $(\mathrm{SD}=1.3)$, respectively, $t(18)=1.544, p=0.14$. Participants were screened for normal or corrected to normal vision and hearing via self-report. Older adults also had no history of neurological diseases, diabetes or coronary disease, and did not take any medication that might have affected cognition. The Mini-Mental State 
Table 2 | Error rates in percent in single-task trials of single-task blocks, single-task trials in mixed blocks (mixed single-task trials), and dual-task trials for the auditory task in older and younger adults across Session 2-21 (older adults) or Session 2-8 (younger adults).

\begin{tabular}{|c|c|c|c|c|c|c|c|}
\hline \multirow[t]{2}{*}{ Task } & \multirow[t]{2}{*}{ Session } & \multicolumn{3}{|c|}{ Older adults } & \multicolumn{3}{|c|}{ Younger adults } \\
\hline & & $\begin{array}{l}\text { Single-task } \\
\text { trials }\end{array}$ & $\begin{array}{l}\text { Mixed single-task } \\
\text { trials }\end{array}$ & $\begin{array}{l}\text { Dual-task } \\
\text { trials }\end{array}$ & $\begin{array}{l}\text { Single-task } \\
\text { trials }\end{array}$ & $\begin{array}{l}\text { Mixed single-task } \\
\text { trials }\end{array}$ & $\begin{array}{l}\text { Dual-task } \\
\text { trials }\end{array}$ \\
\hline \multirow[t]{20}{*}{ Auditory task } & 2 & 10.7 & 13.5 & 16.6 & 4.1 & 3.7 & 6.3 \\
\hline & 3 & 11.5 & 11.4 & 12.3 & 3.3 & 3.4 & 5.8 \\
\hline & 4 & 8.4 & 9.0 & 12.0 & 1.9 & 3.0 & 3.9 \\
\hline & 5 & 6.6 & 7.6 & 10.6 & 3.1 & 2.4 & 5.4 \\
\hline & 6 & 9.3 & 6.9 & 9.8 & 6.1 & 4.5 & 5.7 \\
\hline & 7 & 6.0 & 7.9 & 9.2 & 5.1 & 4.5 & 5.8 \\
\hline & 8 & 7.0 & 5.8 & 7.3 & 3.9 & 3.5 & 5.6 \\
\hline & 9 & 6.7 & 6.7 & 9.8 & & & \\
\hline & 10 & 7.8 & 7.0 & 10.0 & & & \\
\hline & 11 & 8.2 & 6.8 & 8.7 & & & \\
\hline & 12 & 8.2 & 6.8 & 9.2 & & & \\
\hline & 13 & 6.6 & 5.5 & 8.3 & & & \\
\hline & 14 & 7.2 & 8.8 & 9.7 & & & \\
\hline & 15 & 6.9 & 7.1 & 9.8 & & & \\
\hline & 16 & 9.4 & 8.7 & 10.0 & & & \\
\hline & 17 & 1.1 & 1.2 & 1.5 & & & \\
\hline & 18 & 1.1 & 3.3 & 1.7 & & & \\
\hline & 19 & 1.1 & 0.9 & 1.3 & & & \\
\hline & 20 & 1.8 & 4.3 & 3.0 & & & \\
\hline & 21 & 2.7 & 2.5 & 4.1 & & & \\
\hline
\end{tabular}

Session 2-12: Strobach et al. (2012b), Session 13-16: Experiment 1, Session 17-21: Experiment 2.

Table 3 | Age, formal education, general health status, attention performance, non-verbal intelligence, and vocabulary knowledge for older and younger adults; MMSE (mini-mental state examination) scores for older adults only; CFT 20-R, cultural fair intelligence test, WST, Wortschatztest (vocabulary test).

\begin{tabular}{|c|c|c|c|c|c|c|}
\hline & \multicolumn{3}{|c|}{ Older adults, $N=10(N=8)$} & \multicolumn{3}{|c|}{ Younger adults, $\boldsymbol{N}=10$} \\
\hline & $M$ & SD & Range & $M$ & SD & Range \\
\hline Age (in years) & $63.6(63.3)$ & $3.4(3.8)$ & $57-68(57-68)$ & 22.7 & 3.3 & $19-29$ \\
\hline Education (in years) & $18.0(17.2)$ & $3.9(3.9)$ & $13-24(13-24)$ & $14.2^{* *}$ & 1.4 & $13-16.5$ \\
\hline Health status (1-5) & $4.4(4.5)$ & $0.7(0.8)$ & $3-5(3-5)$ & $3.7 \mathrm{~ns}$ & 1.3 & $1-5$ \\
\hline Attention and concentration performance (d2 Test) overall performance & $410.9(400.0)$ & $90.6(96.3)$ & $284-559(299-559)$ & $532.9 * *$ & 80.0 & $410-632$ \\
\hline Concentration performance & $144.5(139.0)$ & $46.3(50.0)$ & $62-212(62-212)$ & $204.1^{* *}$ & 66.1 & $94-279$ \\
\hline Intelligence test (CFT 20-R) IO & $96.4(96.3)$ & $18.0(20.4)$ & $76-134(76-134)$ & $114.2^{*}$ & 15.4 & $80-142$ \\
\hline Vocabulary test (WST) IQ & $114.2(113.8)$ & $8.6(8.4)$ & $97-125(97-125)$ & $107.3 \mathrm{~ns}$ & 8.0 & $92-118$ \\
\hline MMSE (maximum score $=30$ ) & $29.8(29.7)$ & $0.4(0.5)$ & $29-30(29-30)$ & & & \\
\hline
\end{tabular}

${ }^{*} p<0.01,{ }^{*} p<0.05$, ns, non-significant.

Examination (MMSE; Folstein et al., 1975) indicated no impaired cognitive abilities among the older participants $(M=29.8$, $\mathrm{SD}=0.4$, range $=29-30$ ). A handedness test (Oldfield, 1971) indicated that participants in both groups were right-handed.

In order to further characterize the participants, we conducted paper-and-pencil tests on attention performance ( $\mathrm{d} 2$ Test; Brickenkamp and Zillmer, 1998), a non-verbal intelligence test [Culture Fair Intelligence Test (CFT 20-R); Weiß, 2006], and a vocabulary test [Wortschatztest (WST); Anger et al., 1968]. As illustrated in Table 3, performance in the $\mathrm{d} 2$ Test in the overall and concentration scores was higher in younger adults compared with older adults, $t(18)=3.192, p<0.01$ and $t(18)=2.335, p<0.05$, respectively. Similarly, non-verbal intelligence was optimized in younger adults in contrast with older adults, $t(18)=2.373, p<0.05$. The vocabulary test indicated similar vocabulary knowledge in both groups of participants, $t(18)=1.864, p>0.08$; such findings 
demonstrate the typical finding of impaired fluid processing functions but robust crystallized knowledge across aging (e.g., Cavanaugh and Blanchard-Fields, 2006).

\section{Apparatus and stimuli}

Stimuli were presented on a $17^{\prime \prime}$ color monitor that was connected to a Pentium 1 PC. Experiments were carried out using ERTS software (Experimental Runtime System; Beringer, 2000).

A visual and an auditory task were performed. In the visual task, a circle appeared in one of three possible locations on the screen (left, middle, or right). Participants responded manually, indicating the location of the circle with the corresponding index, middle or ring finger of the right hand. The circles were white and were horizontally arranged on a black background on the computer screen. Each circle subtended approximately $2.5 \mathrm{~cm}$ which corresponds to a $2.38^{\circ}$ visual angle, from a viewing distance of $60 \mathrm{~cm}$. Three horizontal white lines served as placeholders at the possible left, middle, and right locations of the screen. The distance between the circles was $1 \mathrm{~cm}$, which corresponded to approximately $0.95^{\circ}$. All circles subtended approximately $8.99^{\circ}$. Responses were recorded with a response board connected to the computer.

On the auditory task, participants verbally responded to one of three possible sine wave tones played on headphones with a sound level of $75 \mathrm{~dB}$. They responded by saying "ONE" to the low frequency tone $(350 \mathrm{~Hz})$, "TWO" to the middle frequency tone $(900 \mathrm{~Hz})$ or "THREE" to the high frequency tone (1,650 Hz; German: "EINS," “ZWEI," and "DREI”). Verbal reactions were recorded with a Sony microphone connected to a voice key.

\section{Procedure and design}

A single-task trial started with three white lines serving as placeholders signaling the beginning of a trial for $500 \mathrm{~ms}$. After this period had elapsed, an additional circle appeared in the visual task and remained visible until the participant responded or until a maximum of 2,000 ms had elapsed. A tone lasting for $40 \mathrm{~ms}$ was played in the auditory task. In dual-task trials, a circle and a tone were presented simultaneously. RTs were given as feedback after each trial for $1,500 \mathrm{~ms}$ followed by a blank screen for $700 \mathrm{~ms}$. In dual-task trials, only the faster of the two RTs was given as feedback at the end of the trial to minimize the load. When participants committed an error or 2,000 ms had elapsed, the RT feedback was replaced by the German word for error ("Fehler") for the same amount of time.

There were two types of blocks: single-task blocks and mixed blocks. In the single-task blocks, participants performed either 45 single-task trials of the visual task or of the auditory task. During mixed blocks, participants performed a mixture of 30 single-task trials (mixed single-task trials), 15 of the visual task and 15 of the auditory task, and 18 dual-task trials. All trials were randomly intermixed, requiring participants to switch between processing different single-task and dual-task trials. Participants were instructed to respond to both stimuli as quickly and accurately as possible during all blocks, to give these their full concentration and to give both tasks equal priority.
In order to familiarize the participants with the characteristics of the visual and auditory task and so that these could learn these tasks before presenting dual-task trials, participants exclusively performed six visual and six auditory single-task blocks in Session 1; these blocks were presented in an alternating order. Session 2 included six single-task blocks (three visual and three auditory single-task blocks) and eight mixed blocks. After two initial single-task blocks (one visual and one auditory single-task block), sequences of two mixed blocks and one single-task block followed in this session. The design in Sessions 3-16 was identical to that in Session 2 but these sessions included two additional mixed blocks at the end. In the Sessions 2-16, half of the participants always started with a visual single-task block and the other half always with an auditory single-task block; subsequently, the type of single-task block (i.e., visual or auditory) alternated. While Session 1 lasted around $45 \mathrm{~min}$ the following sessions took about $60 \mathrm{~min}$. Sessions were conducted on successive days (excluding weekends). In this way, all sessions were completed within 2 weeks.

To maximize participants' motivation for achieving fast performance, reward was given in the form of a monetary performancebased payoff (see also Schumacher et al., 2001; Tombu and Jolicoeur, 2004). The payoff matrix was based on an adaptive comparison between participant's RT in a current block and a reference RT; this reference RT represents the individual best mean block RT and is adjusted separately for the visual and the auditory task and for task conditions (single-task trials in single-task blocks vs. dualtask trials). Participants could earn the more money the nearer the current RTs were to the reference RTs or if current block RTs were faster than the reference RTs; in the latter case reference RTs were adjusted to current block RTs. Bonus payments were also made on the basis of accuracy rates: A bonus was given for each correct response while there was a deduction from this bonus for each incorrect response.

\section{RESULTS \\ RT analyses}

For the analysis of the training effects in the older adults, we compared the data of the final training session before the present training phase started (i.e., Session 12 of Strobach et al., 2012b) with the final session in the present training phase (i.e., Session 16). Therefore, we conducted $2 \times 3$ mixed measures ANOVAs with the within-subject factors SESSION (Session 12 vs. 16) and TRIALTYPE (single-task trials, mixed single-task trials, and dual-task trials) separately for each component task. Following the RT training data, we analyzed the performance in older adults' Session 16 and younger adults' Session 8. Our primary indicator of dualtask performance was the RT difference between dual-task trials and mixed single-task trials that reflects dual-task costs. In addition, we report the difference between mixed single-task trials and single-task trials that reflects task-set costs; this measure demonstrates the requirement to prepare for and maintain multiple task sets in mixed single-task conditions as compared with the condition of single-task blocks (Rogers and Monsell, 1995; Kray and Lindenberger, 2000; Bherer et al., 2005).

During older adults' training, RTs in the visual task declined considerably, $F(1,9)=11.041, p<0.01$, partial $\eta_{p}^{2}=0.55$. In 
addition, RTs differed between trial types, $F(2,18)=19.420$, $p<0.001$, partial $\eta_{\mathrm{p}}^{2}=0.68$, indicating higher RTs in dual-task trials followed by mixed single-task trials and single-task trials (dual-task vs. mixed single-task trials and mixed single-task trials vs. single-task trials: all $p s<0.05)$. TRIALTYPE was qualified by an interaction with SESSION, $F(2,18)=10.310, p<0.001$, partial $\eta_{\mathrm{p}}^{2}=0.53$. A decomposition of this interaction into comparisons of mixed single-task trials vs. dual-task trials and single-task trials vs. mixed single-task trials in Session 12 and 16 showed that dual-task costs, $F(1,9)=9.558, p<0.05$, partial $\eta_{\mathrm{p}}^{2}=0.52$, and task-set costs, $F(1,9)=9.292, p<0.05$, partial $\eta_{\mathrm{p}}^{2}=0.51$, decreased during training (Figure 1). The comparison of the dual-task performance of younger adults in Session 8 and this performance of older adults in Session 16 showed larger dual-task costs in the latter group [older adults: $43 \mathrm{~ms}, t(9)=3.484, p<0.01$; younger adults: $15 \mathrm{~ms}, t(9)=3.815, p<0.01$; between group comparison: $F(1,18)=8.022, p<0.01$, partial $\eta_{\mathrm{p}}^{2}=0.31$ ] while task-set costs showed no statistical group difference [older adults: $10 \mathrm{~ms}, t(9)=3.550, p<0.01$; younger adults: $12 \mathrm{~ms}, t(9)=2.955$, $p<0.05$; between group comparison: $F(1,18)<1$ ] as illustrated in Figure 2.

In the auditory task, RTs were slower in Session 12 than in Session 16, $F(1,9)=33.333, p<0.001$, partial $\eta_{\mathrm{p}}^{2}=0.79$. Also, RTs were slower in dual-task trials than in mixed singletask trials $(p<0.001)$ followed (by trend) in single-task trials $(p<0.077), F(2,18)=17.457, p<0.001$, partial $\eta_{\mathrm{p}}^{2}=0.66 . \mathrm{A}$ non-significant interaction of SESSION and TRIALTYPE, $F(6$, $54)=1.897, p>0.179$, partial $\eta_{p}^{2}=0.17$, indicated similar training effects on all types of trials. Similar to the visual task, we found increased dual-task costs in older adults' Session 16 when contrasted with these costs of younger adults in Session 8 [older adults: $57 \mathrm{~ms}, t(9)=4.889, p<0.001$; younger adults: $22 \mathrm{~ms}, t(9)=4.787, p<0.001$; between group comparison: $F(1$, $18)=8.022, p<0.01$, partial $\eta_{\mathrm{p}}^{2}=0.31$ ] while task-set costs showed no group difference [older adults: $20 \mathrm{~ms}, t(9)=1.935$,

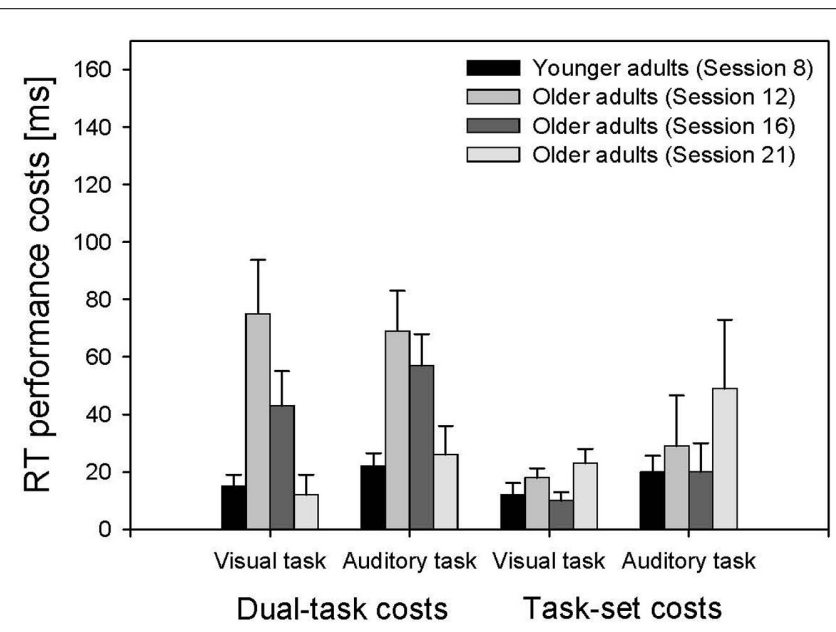

FIGURE 2 | Dual-task and task-set costs in younger adults (Session 8) and older adults (Session 12, 16, 21) in RTs. Session 8 and 12: Strobach et al. (2012b), Session 16: Experiment 1, Session 21: Experiment 2. $p>0.08$; younger adults: $20 \mathrm{~ms}, t(9)=3.529, p<0.01$; between group comparison: $F(1,18)<1$ ] as illustrated in Figure 2 .

In the preceding analyses we used a strong and reliable criterion for measuring dual-task performance, by assessing dual-task costs in the RT comparison of dual-task trials and mixed single-task trials (i.e., dual-task costs = dual-task RTs - mixed single-task RTs; Bherer et al., 2006; Hazeltine et al., 2002). However, this criterion may lead to interpretative difficulties if there were baseline differences in performance due to the general slowing of processing in older adults (Somberg and Salthouse, 1982; Guttentag, 1989; Riby et al., 2004); in fact, this might have obscured possible differences between younger adults' dual-task performance in Session 8 and older adults' dual-task performance in Session 16 in the visual and auditory RT data. Therefore, we additionally assessed dual-task performance in terms of proportional dual-task costs to control for baseline differences between the age groups: proportional dual-task costs $=($ dual-task RTs - mixed single-task RTs $) /$ mixed single-task RTs (Riby et al., 2004). The analyses of proportional dual-task costs corroborated the findings in the analyses of dualtask costs: Older adults showed larger proportional dual-task costs in the visual task, $t(18)=2.174, p<0.05$, and the auditory task, $t(18)=2.429, p<0.05$, than younger adults at the end of training. Thus, the appearance of dual-task cost differences between both aging groups is not confounded by possible differences in singletask performance between groups; therefore a general slowing in older adults cannot explain the observed differences in dual-task costs of Session 16 (older adults) and Session 8 (younger adults; Verhaeghen et al., 2003).

In addition, we analyzed whether the mean dual-task advantage in younger adults compared with older adults also holds at an individual level of data analysis (Schumacher et al., 2001; Hartley et al., 2011). For this purpose, we plotted the dual-task costs of the visual and the auditory task for each individual older and younger adult in Session 8 and 16, respectively (Figure 3). In this Brinley plot, data points for individual participants with lower costs in both tasks are located in the lower left corner while participants with larger costs are located in the upper right corner. Data points for younger adults are mostly in the lower left corner that represents relatively low dual-task costs of both tasks in Session 8. Data points of most individual older adults are at positions that represent larger costs and impaired dual-task performance relative to younger adults. Only some older adults showed data at a performance level approaching that of the younger adults. Thus, the observed difference in mean dual-task costs between the age groups at the end of training, therefore largely holds at an individual level.

Summarizing the RT data, older adults showed a benefit of training on dual-task and task-set costs from Session 12 to 16 in the visual task, but not in the auditory task. Important for the present question of near perfect time sharing, dual-task costs in older adults were still increased after doubling the number of their training sessions compared to younger adults. Task-set costs were similar across both age groups.

\section{Error analyses}

Similar to the training RT data, error rates in Session 12-16 were analyzed for single-task, mixed single-task, and dual-task trials 


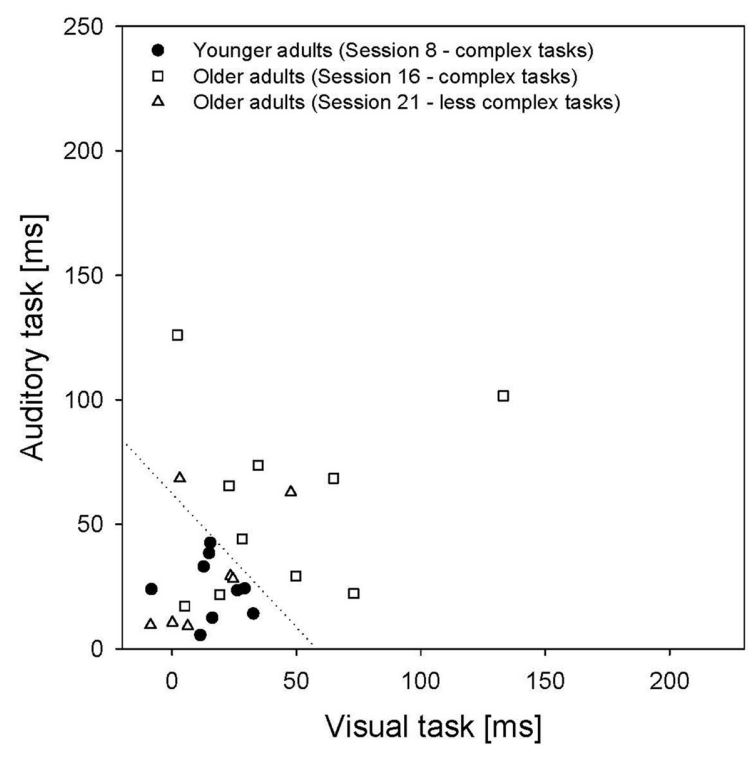

FIGURE 3 | Individual dual-task reaction time (RT) costs in younger and older adults at the end of practice (i.e., younger adults: Session 8, older adults: Session 16, 21). The $x$-axis represents the costs in the visual task while the $y$-axis represents the costs in the auditory task.

in older adults. In the visual task, there were lower error rates in dual-task than in mixed single-task, and in single-task trials, $F(2,18)=9.509, p<0.01$, partial $\eta_{p}^{2}=0.51$ (dual-task vs. mixed single-task trials and mixed single-task trials vs. single-task trials: all $p s<0.05$; Table 1). The effect of SESSION were marginally significant, $F(1,9)=5.102, p=0.05$, partial $\eta_{\mathrm{p}}^{2}=0.36$, revealing increased error rates in Session 16 than in Session 12. The interaction of both factors was non-significant, $F(2,18)=1.274, p>0.31$, partial $\eta_{\mathrm{p}}^{2}=0.12$. The findings of increased single-task error rates and increased error rates at the end of training are consistent with previous findings in younger adults using a similar task situation (Schumacher et al., 2001; Hazeltine et al., 2002; Tombu and Jolicoeur, 2004; Liepelt et al., 2011; Strobach et al., in press) and may be explained by a reduced degree of attentiveness in the visual task due to reduced processing demands. The analysis of older adults' error rates during training revealed no effects or interaction in the auditory task (Table 2).

\section{DISCUSSION}

In Experiment 1, we tested whether an increased amount of dualtask training in older adults (i.e., 16 sessions) improved dual-task performance in these learners to a level of near perfect time sharing in younger adults with only eight training sessions. The analyses of mean RT data revealed that older adults still show increased dual-task costs. Thus, this group did not optimize dual-task performance to a level achieved by younger adults. This difference even remained after we controlled for baseline differences in processing speed (i.e., proportional dual-task costs; e.g., Riby et al., 2004). Observations on a participant level demonstrated that most individual older adults performed on a lower dual-task level than the individuals of the younger adult group.
Could it be that older adults simply need even more training sessions to arrive at the same level of dual-task performance as younger adults? Generally, there is no way to rule out this conjecture; for any finite amount of training given to older adults, the possibility remains that more training would eventually eliminate dual-task cost differences between groups. However, we believe that moderately more training for older adults would not have changed the results concerning the level of dual-task performance because training had no RT effect on the dual-task costs of the visual and the auditory task, $F \mathrm{~s}(1,9)<1$, across the last two training sessions. That is, the dual-task costs of the older adults were not further reduced at the end of training in Experiment 1 .

Alternative to an increased amount of training, older adults may achieve the level of near perfect time sharing of younger adults due to training with component tasks that are simpler when compared to the tasks applied in Experiment 1. We tested this assumption in the following Experiment 2.

\section{EXPERIMENT 2}

A number of dual-task studies reported that simplified component tasks lead to a reduced impairment of one or both tasks in dual-task situations and, therefore, a reduction of dual-task costs (e.g., Frith and Done, 1986; Pashler, 1994; Van Selst and Jolicoeur, 1997; Schubert, 1999, 2008). This was particularly demonstrated for practiced dual-task performance in older adults (Maquestiaux et al., 2004).

One way to simplify tasks and, as a result, optimize dual-task performance/reduce dual-task costs is to reduce the number of stimulus-response mappings in the component tasks when contrasted with more difficult tasks. There exist two sources to explain reduced dual-task costs in situations with tasks that include a reduced number of stimulus-response mappings. First, the number of these mappings particularly affects the processing time of a central response selection stage (e.g., Schubert, 1999). When the processing time of the response selection stage is shortened, the likelihood of an overlap of the potential capacity-limited bottleneck stages in two concurrent tasks is reduced; in this way, the interference between concurrently presented tasks and the resulting dual-task costs are reduced. An additional source to explain reduced dual-task costs with tasks including a reduced number of stimulus-response mappings is associated with task coordination processes (Logan and Gordon, 2001). For instance, such processes perform a switch between capacity-limited central stages in a first task and in a second executed task (Maquestiaux et al., 2004; Sigman and Dehaene, 2006; Liepelt et al., 2011). Operations carried out during these processes potentially include activating or instantiating the stimulus-response mapping rules of the second task. To do so, it may be that the rules must be reactivated in or moved back into workingmemory. The important point is that this switching stage functions more efficiently with a reduced amount of information handled in the case of simpler component tasks. This efficient functioning may result in faster switching between tasks and in a reduction of dual-task costs; previous findings of Maquestiaux et al. (2004) are consistent with this assumption. Taken together, we hypothesize that the reduction of the number of mapping rules may lead to shortened bottleneck stages within 
the component tasks and/or facilitate a switching process in older adults which may lead to reduced age differences in dual-task performance.

To investigate the effects after dual-task training with simplified component tasks, the older adults of Experiment 1 continued training after changing from three-choice to two-choice versions of the visual and the auditory tasks. In these two-choice versions, the visual task exclusively included presentations of circles at a left or a right position while low and high tones were exclusively presented in the auditory task. These tasks were trained for five sessions; note that we refer to these sessions in the following sections of Experiment 2 as Sessions 17-21, as older adults continued this training after the end of training in Session 1-16 (Strobach et al., 2012b; the present Experiment 1). The performance in the older adults' Session 21 was compared with performance of near perfect time sharing in younger adults after eight training sessions. If training with simplified tasks produces conditions for near perfect time sharing in older adults, we expect the same level of dual-task performance across the included age groups, i.e., similarly reduced dual-task costs in younger and older adults. However, if training with such simplified tasks is not sufficient to produce conditions for near perfect time sharing in older adults, dual-task costs should not be reduced in this age group and should increase the amount of younger adults' costs in Session 8.

\section{METHOD}

\section{Participants}

The groups of older and younger participants were identical to Experiment 1 with the exception that two older adults (one female, one male) were not available for further training sessions. As illustrated in Table 3, age, formal education, general health status, attention and concentration performance, nonverbal intelligence, vocabulary knowledge, and MMSE scores for the remaining older adults (in brackets) were similar to these data in Experiment 1. In addition, for the data in Session 16, we found similar dual-task RT and error costs in the older adults included into Experiment 2 when compared to that group of older adults in Experiment $1, F s(1,16)<1$. So, participants in Experiment 2 are highly representative for the group of older adults in Experiment 1 .

Apparatus, stimuli, procedure, and design in Session 17-21 were identical to Experiment 1 with the following exceptions. In the visual task, circles exclusively appeared at the left or the right location on the screen while participants responded to one of only two possible tones, the low frequency and the high frequency tones. Single-task blocks included 46 single-task trials while mixed blocks included 52 trials (20 dual-task trials, 16 visual single-task trials, 16 auditory single-task trials); the trial number in singletask and mixed blocks was varied after Experiment 1 to fit the requirement of a similar proportion of single and dual-task trials with three-choice and two-choice tasks across Experiment 1 and 2 , respectively.

\section{RESULTS}

The data handling for statistical analysis was similar to Experiment 1.

\section{RT analyses}

In older adults, training RTs in the visual task differed between trial types, $F(1,7)=19.152, p<0.001$, partial $\eta_{\mathrm{p}}^{2}=0.73$, indicating higher RTs in dual-task trials followed by mixed single-task trials, and single-task trials (dual-task vs. mixed single-task trials and mixed single-task trials vs. single-task trials: all $p s<0.01$ ). The effect of SESSION, $F(1,7)=46.555, p<0.001$, partial $\eta_{\mathrm{p}}^{2}=0.87$, revealing faster RTs in Session 21 than in Session 16, was modulated by TRIALTYPE, $F(2,14)=4.313, p<0.05$, partial $\eta_{\mathrm{p}}^{2}=0.38$. A decomposition of this modulation into comparisons of mixed single-task trials vs. dual-task trials and single-task trials vs. mixed single-task trials in Session 16 and 21 demonstrated that dualtask costs, $F(1,7)=7.896, p<0.05$, partial $\eta_{\mathrm{p}}^{2}=0.53$, decreased and task-set costs, $F(1,7)=14.785, p<0.01$, partial $\eta_{\mathrm{p}}^{2}=0.70$, increased during training (Figure 1). Importantly, the comparison of the dual-task performance of younger adults in Session 8 and this performance in older adults in Session 21 revealed no difference in dual-task costs [older adults: $12 \mathrm{~ms}, t(7)=1.831$, $p>0.10$; younger adults: $15 \mathrm{~ms}, t(9)=3.815, p<0.01$; between group comparison: $F(1,16)<1]$ as well as in proportional dualtask costs, $t(16)<1$. Further, there was no statistical group difference in the task-set costs [older adults: $23 \mathrm{~ms}, t(7)=4.489$, $p<0.05$; younger adults: $12 \mathrm{~ms}, t(9)=2.955, p<0.05$; between group comparison: $F(1,18)=2.851, p>0.11$ ] as illustrated in Figure 2.

The auditory task RT data showed faster responses in Session 21 than in Session 16, $F(1,7)=18.079, p<0.01$, partial $\eta_{p}^{2}=0.72$. Also, RTs were faster in single-task, than in mixed single-task, and in dual-task trials, $F(2,14)=15.754, p<0.001$, partial $\eta_{\mathrm{p}}^{2}=0.69$ (dual-task vs. mixed single-task trials and mixed single-task trials vs. single-task trials: all $p s<0.05)$. TRIALTYPE was qualified by an interaction with SESSION, $F(2,14)=6.271, p<0.05$, partial $\eta_{\mathrm{p}}^{2}=0.47$. As illustrated in Figure 1, the magnitude of dual-task costs decreased, $F(1,7)=4.810, p<0.05$, partial $\eta_{\mathrm{p}}^{2}=0.42$, while task-set costs increased from Session 16 to $21, F(1,7)=21.642$, $p<0.01$, partial $\eta_{\mathrm{p}}^{2}=0.76$; these latter findings parallel the progression of these costs in the visual task. Also similar to the visual task, we found no difference between the dual-task costs in the older adults' Session 21 and younger adults' Session 8 [older adults: $26 \mathrm{~ms}, t(7)=2.610, p<0.05$; younger adults: $22 \mathrm{~ms}, t(9)=4.787$, $p<0.001$; between group comparison: $F(1,16)<1$ ] as well as no difference in the proportional dual-task costs, $t(16)=1.941$, $p>0.09$. Task-set costs were increased in older when compared to younger adults in these sessions [older adults: $49 \mathrm{~ms}, t(7)=4.667$, $p<0.01$; younger adults: $20 \mathrm{~ms}, t(9)=3.529, p<0.01$; between group comparison: $F(1,18)=6.981, p<0.05$, partial $\eta_{p}^{2}=0.30$ ] as illustrated in Figure 2; the latter may result from an increased training benefit of single-task compared with mixed single-task trials in older adults.

Illustrations of individual dual-task costs in the visual and auditory task revealed that after additional training with simplified tasks, most of the older adults reached the dual-task performance level of younger adults in Session 8 (Figure 3).

Summarizing the RT data, older adults showed a training effect on dual-task and task-set costs in both tasks. Most important for the question about limits of dual-task performance in older adults, we showed similarly reduced dual-task costs, i.e., near perfect time 
sharing, in older and younger adults at the end of training (i.e., Session 21 in older adults vs. Session 8 in younger adults).

\section{Error analyses}

In the training data of the visual task (Table 1), TRIALTYPE was significant, $F(2,14)=3.962, p<0.05$, partial $\eta_{p}^{2}=0.36$, demonstrating lower error rates in dual-task than in single-task trials $(p<0.05)$; this dual-task advantage in the error rates of the visual task is well-known from previous studies (e.g., Hazeltine et al., 2002; Strobach et al., in press) and the present Experiment 1. There was no effect of, or interaction with SESSION. The analysis of error rates in the auditory task revealed no effects or interaction (Table 2).

\section{DISCUSSION}

The findings of the visual and the auditory task demonstrate near perfect time sharing at the end of training in older adults (Session 21 ) and younger adults (Session 8). In fact, absolute and proportional dual-task costs were similar in both groups. Note that this similar cost level was only achieved with additional training and simpler component tasks in older adults relative to younger adults. The analysis of individual dual-task costs largely confirmed the analysis at the group level.

The dual-task performance of older adults in Session 21 represented the first session, in which these participants showed the same optimized dual-task "target" performance for both tasks that younger adults showed at the end of their training. In fact, absolute dual-task costs in the visual task were similar in older and younger adults already prior to Session 21 (i.e., Session $17-20)$, $t \mathrm{~s}(16)<2.052$, $p s>0.06$, but they were larger for older adults in the auditory task in all prior sessions, $t \mathrm{~s}(16)>2.289$, $p s<0.05$. Thus, the combination of the training Sessions 17-21 and reduced task complexity (after the completion of prior 16 sessions with more complex tasks) is essential for optimized dual-task performance in older adults.

\section{GENERAL DISCUSSION}

The aim of the present study was to test the limits of optimized dual-task performance in older adults (i.e., Baltes and Kliegl, 1992) through the application of appropriate conditions for such optimization. Based on a dual-task situation including conditions for near perfect time sharing in younger adults (Schumacher et al., 2001; Strobach et al., 2012b), we tested whether an increased amount of training in this situation and/or training in this situation with simplified component tasks represent such conditions for older adults.

A basic finding of the present study is that older adults demonstrated improved dual-task performance with practice; this improvement parallels findings of a number of previous dualtask practice studies in this age group (e.g., Maquestiaux et al., 2004; Allen et al., 2009; Hartley et al., 2011). A novel finding of the present study is, however, that older adults even improve dual-task performance after extensive prior training over 12 sessions. This finding shows the substantial plasticity in cognitive functioning in this age group (Kramer and Willis, 2003) on the one hand; on the other hand, one may suggest that the implementation of this plasticity requires a large amount of practice.
Concerning our primary focus on the limits of dual-task optimization in older adults, our results show that an increased amount of training in older compared with younger adults does not result in optimized dual-task performance. That is, the dualtask costs of older adults were increased compared to these costs in younger adults in Experiment 1. However, after training with simpler component tasks in older adults, we observed similar levels of dual-task performance across different age groups. These similar levels in younger and older adults are indicated by similar dual-task costs in Session 21 (older adults) and Session 8 (younger adults) of Experiment 2. In this way, older adults as well as younger adults achieved near perfect time sharing ${ }^{1}$.

However, the achievement of similar dual-task performance levels in older adults, compared with younger adults, occurs exclusively under very specific conditions. First, we tested dual-task performance under conditions that were defined as optimal for younger adults (e.g., Meyer and Kieras, 1999); these findings are reported in Strobach et al. (2012b). Second, we doubled the amount of training of older compared to younger adults under these optimal dual-task conditions. Third, we continued to adapt these conditions to the requirements of older adults through the introduction of simpler component tasks in this age group. The dual-task performance in older adults exclusively adjusted to near perfect time sharing under the latter condition.

From a different perspective, one may critically argue that these specific conditions of testing older adults' dual-task performance were unfair when faced with the test conditions in younger adults. We do not disagree with this argument. However, investigating the effects of training on dual-task performance under identical conditions was not the critical issue of the present study. Instead, we aimed to achieve near perfect time sharing in older adults (i.e., the developmental reserve). Testing the developmental reserve under identical conditions in younger and older adults was the aim of Bherer et al. (2006). Similar to the findings of Strobach et al. (2012b), these authors demonstrated similar effects across an identical amount of training in these age groups. Bherer et al. $(2005,2006)$ as well as Strobach et al. demonstrated still increased dual-task performance costs at the end of this training in older compared with younger adults. These findings were consistent although both lines of studies (i.e., Bherer and colleagues/Strobach

\footnotetext{
${ }^{1}$ An increased amount of education in older compared with younger adults (see Table 3) had no impact on the between group comparisons (i.e., older vs. younger adults) of dual-task and task-set costs at the end of training in Experiment 1 and 2. These comparisons were similar in analyses of the visual and auditory task when years of education were introduced as a covariate into the mixed measures ANOVAs including the factors SESSION, AGE GROUP, and TRIALTYPE (dual-task costs: dual-task trials vs. mixed single-task trials; task-set costs: mixed single-task trials vs. single-task trials) and with no covariate inclusion into such analyses (see Results). This finding is consistent with a comparison between older and younger adults at the end of the same amount of training in both age groups (i.e., Session 8, Strobach et al., 2012b). Furthermore, impacts of fatigue and/or training did not obscure the between group comparisons of dual-task and task-set costs within Session 16 (Experiment 1) and 21 (Experiment 2). This was demonstrated by non-significant effects or interactions of the additional factor PHASE (first session half vs. second session half) in mixed measures ANOVAs including the factors AGE GROUP and TRIALTYPE (dual-task costs: dual-task trials vs. mixed single-task trials; task-set costs: mixed single-task trials vs. single-task trials) on the visual and auditory task data in Session 16 and 21.
} 
and colleagues) applied component tasks with different levels of complexity (two-choice vs. three-choice RT tasks) and different output-modality combinations (two manual tasks vs. one manual/one vocal task).

However, training with one manual and one vocal task exclusively under a two-choice condition (with extensive prior training with three-choice tasks) enables near perfect time sharing in older adults, as illustrated in the present Experiment 2. Based on the present training design, we cannot conclusively disentangle whether the critical factor for such performance is the extensive prior training (reported in Strobach et al., 2012b, plus the present Experiment 1) and/or the introduction of simplified tasks. We assume however that extensive training does not explain near perfect time sharing exclusively and task simplification is the major factor leading to this performance level. This assumption is supported by our observation of no further training benefit at the end of 16 sessions with the three-choice RT tasks (see Discussion of Experiment 1) but a continuation of this benefit with two-choice RT tasks in the subsequent sessions. The exclusive impact of prior training on near perfect time sharing in the present Experiment 2 is further weakened by the findings of Maquestiaux et al. (2004) demonstrating a reduced training benefit in older adults with complex tasks and a following impressive drop of dual-task costs (in their case the psychological refractory period effect; Pashler, 1994) in older compared with younger adults after the introduction of simplified component tasks. (Unfortunately, there was no subsequent training in the dual-task situation with the simplified tasks to test its training effect and to provide conclusions about the impact of prior training and task simplification on near perfect time sharing in Maquestiaux et al.) Potentially, the introduction of two-choice RT tasks allows for a continuation of the dual-task performance improvement to the level of near perfect time sharing because both of these simpler tasks could be efficiently activated in working-memory, while this task activation is not efficient with more complex tasks (i.e., three-choice RT tasks). Such non-efficient activation may result from impaired workingmemory functions particularly present in older adults (Hartley and Little, 1999; Maquestiaux et al., 2004). Due to the equal priority instructions on both tasks of our dual-task situation as well as feedback and monetary bonuses in all sessions, there was no assessment of the testing the limits parameters baseline (i.e., standard conditions) and baseline reserve (i.e., optimized standard conditions due to, for instance, motivation). Therefore, there are no conclusions regarding these parameters from the present study in comparison to their outcomes in Bherer et al. (2006).

In a recent study, Hartley et al. (2011) provided evidence that some older adults showed performance consistent with perfect time sharing and did so with relatively little training. However, "compared to the central processes required in the conventional dual-task procedure, this (i.e., Hartley et al.'s) procedure reduced the demands of stimulus categorization" (p.186) by perfect redundancy between the stimuli of two tasks in dual-task situation (e.g., a left circle in a visual task was always combined with a low tone in an auditory task). Therefore, we assume no performance of two completely unrelated component tasks in the dual-task situation of Hartley and colleagues. Consequently, we present the first study in the aging literature that achieved optimized performance (i.e., near perfect time sharing) in a "conventional" dual-task situation.

However, we demonstrated findings of near perfect time in a dual-task situation that showed even zero dual-task costs (i.e., perfect time sharing) in a similar dual-task situation. That is, at the end of training, RT differences between dual-task and single-task trials were greatly reduced, but residual dual-task costs remained even in younger adults. This suggests that findings of a complete dual-task cost reduction are not easily obtained as a result of dual-task training (Schumacher et al., 2001), which is in line with a range of previous findings (Hazeltine et al., 2002; Tombu and Jolicoeur, 2004). The finding of residual dual-task costs in the present study might be due to the use of separate deadlines for dual-task and single-task conditions taken as the basis of the financial payoff matrix. This procedure might maintain strong and equal motivation for both single-task trials and dual-task trials until the end of training (Tombu and Jolicoeur, 2004). In contrast, Schumacher et al. (2001) exclusively used the performance deadline of the single-task trials presented during the mixed blocks to award financial payoff in both single-task and dual-task trials during training (see also Hazeltine et al., 2002). The Schumacher procedure might increase effects of mobilized effort in dual-task trials as compared to single-task trials. As a result of these unequal effects, one should find a greater reduction of RTs in dual-task than in single-task during training. This difference in deadline procedures between studies might explain the finding of non-significant dual-task costs in the study by Schumacher and colleagues (i.e., perfect time sharing) in contrast to the small residual dual-task costs we found at the end of training (i.e., near perfect time sharing).

In the terminology of the testing the limits approach, we provided evidence that, under very specific conditions, the developmental reserve of older adults enables optimized dual-task performance. Although further studies are needed to better understand how and when age impairs the ability to perform concurrent tasks, the results reported here, along with previous training studies (e.g., Maquestiaux et al., 2004; Allen et al., 2009; Hartley et al., 2011), suggest that the ability to dual-task can be substantially improved in older adults. Within the context of the testing the limits approach, our results suggest that age does not necessarily reduce the range of cognitive plasticity that can be achieved after substantial training. One open question of the present study, however, refers to the limits of cognitive plasticity and optimized dual-task performance in old-older adults; note that we included relatively young older adults in the present study who were largely in their sixties. From studies on other cognitive domains, it is known that with increasing age, adults are less likely to efficiently use newly acquired skills and strategies (e.g., Nyberg et al., 2003; Buschkuehl et al., 2008); thus, more elderly adults may not benefit from the present type of dual-task training to the extent of older adults. Another open question refers to the underlying practicerelated mechanisms of optimized dual-task performance in older adults. These mechanisms may be associated with either processing changes within the component tasks that constitute a dual-task situation (e.g., Ruthruff et al., 2006; Maquestiaux et al., 2010) or the acquisition of improved task coordination skills in older adults. Particularly, the latter option is of interest as there exist opposing 
theoretical assumptions in the aging literature that are consistent (e.g., Hirst et al., 1980; Kramer et al., 1995; Bherer et al., 2005) or inconsistent (e.g., Maquestiaux et al., 2004) with such a skill acquisition.

\section{CONCLUSION}

Older adults are able to improve dual-task performance even after they have already conducted extensive prior training. Under very specific conditions (i.e., training with simplified component tasks), this age group demonstrates a similar level of optimized dual-task performance when contrasted with that performance in younger adults, i.e., near perfect time sharing. In this way, we

\section{REFERENCES}

Allen, P. A., Ruthruff, E., Elicker, J. D., and Lien, M. (2009). Multisession, dual-task psychological refractory period practice benefits older and younger adults equally. Exp. Aging Res. 35, 369-399.

Allen, P. A., Smith, A. F., Vires-Collins, H., and Sperry, S. (1998). The psychological refractory period: evidence for age differences in attentional time-sharing. Psychol. Aging 13, 218-229.

Anger, H., Hylla, E., Horn, H., Schwarz, E., Raatz, U., and Bargmann, R. (1968). Wortschatztest WST 7-8. Begabungstest für 7. und 8. Klassen. Weinheim: Verlag Julius Beltz.

Baltes, P. B., and Kliegl, R. (1992). Further testing of limits of cognitive plasticity: negative age differences in a mnemonic skill are robust. Dev. Psychol. 28, 121-125.

Beringer, J. (2000). Experimental Runtime System. Frankfurt am Main: BeriSoft Cooperation.

Bherer, L., Kramer, A. F., Peterson, M. S., Colcombe, S., Erickson, K., and Becic, E. (2005). Training effects on dual-task performance: are there age-related differences in plasticity of attentional control? Psychol. Aging 20, 695-709.

Bherer, L., Kramer, A. F., Peterson, M. S., Colcombe, S., Erickson, K., and Becic, E. (2006). Testing the limits of cognitive plasticity in older adults: application to attentional control. Acta Psychol. (Amst.) 123, 261-278.

Bherer, L., Kramer, A. F., Peterson, M. S., Colcombe, S., Erickson, K., and Becic, E. (2008). Transfer effects in task-set cost and dual-task cost after dual-task training in older and younger adults: further evidence for cognitive plasticity in attentional control in late adulthood. Exp. Aging Res. 34, 188-209.

Brickenkamp, R., and Zillmer, E. (1998). The d2 Test of Attention. (1st US $E d n$.). Seattle, WA: Hogrefe and Huber Publishers.

Buschkuehl, M., Jaeggi, S. M., Hutchison, S., Perrig-Chiello, P., Däpp, C.,
Müller, M., and Perrig, W. J. (2008). Impact of working memory training on memory performance in old-old adults. Psychol. Aging 23, 743-753.

Cavanaugh, J. C., and BlanchardFields, F. (2006). Adult Development and Aging, 5th Edn. Belmont, CA: Wadsworth Publishing/Thomson Learning.

Folstein, M. F., Folstein, S. E., and McHugh, P. R. (1975). "Mini mental state" - a practical method for grading the cognitive state of patients for the clinician. J. Psychiatr. Res. 12, 189-198.

Frith, C. D., and Done, D. (1986). Routes to action in reaction time tasks. Psychol. Res. 48, 169-177.

Glass, J. M., Schumacher, E. H., Lauber, E. J., Zurbriggen, E. L., Gmeindl, L., Kieras, D. E., and Meyer, D. E. (2000). Aging and the psychological refractory period: taskcoordination strategies in young and old adults. Psychol. Aging 15, 571-595.

Göthe, K., Oberauer, K., and Kliegl, R. (2007). Age differences in dual-task performance after practice. Psychol. Aging 22, 596-606.

Guttentag, R. E. (1989). Age differences in dual-task performance: procedures, assumptions, and results. Dev. Rev. 9, 146-170.

Hartley, A. A. (2001). Age differences in dual-task interference are localized to response-generation processes. Psychol. Aging 16, 47-54.

Hartley, A. A., and Little, D. M. (1999). Age-related differences and similarities in dual-task interference. J. Exp. Psychol. Gen. 128, 417-450.

Hartley, A. A., Maquestiaux, F., and Silverman Butts, N. (2011). A demonstration of dual-task performance without interference in some older adults. Psychol. Aging 26, 181-187.

Hazeltine, E., Teague, D., and Ivry, R. B. (2002). Simultaneous dual-task performance reveals parallel response selection after practice. J. Exp. Psychol. Hum. Percept. Perform. 28, 527-545.

tested the performance limits of older adults in dual-tasks and the cognitive plasticity associated in performing these situations.

\section{ACKNOWLEDGMENTS}

This research was supported by a grant from the German Research Foundation to Torsten Schubert, Peter Frensch, and Herrmann Josef Müller (DFG Schu 1397/5-1; DFG Fr 1493/3-2) as well as by the ESCoP Early Career Stimulus to Tilo Strobach. We would like to thank Julia Steudte, Christina Reimer, Tobias Baumann, and Dejan Draschkow for their assistance with data collection. We thank two the reviewers for their helpful comments on an earlier version of the manuscript.

Hein, G., and Schubert, T. (2004) Aging and input processing in dualtask situations. Psychol. Aging 19, 416-432.

Hirst, W., Spelke, E. S., Reaves, C. C., Caharack, G., and Neisser, U. (1980). Dividing attention without alteration or automaticity. J. Exp. Psychol. Gen. 109, 98-117.

Kramer, A. F., Larish, J. F., and Strayer, D. L. (1995). Training for attentional control in dual task settings: a comparison of young and old adults. $J$. Exp. Psychol. Appl. 1, 50-76.

Kramer, A. F., and Willis, S. L. (2003). "Cognitive plasticity and aging," in The Psychology of Learning and Motivation: Advances in Research and Theory, Vol. 43, eds B. H. Ross and B. H. Ross (New York: Elsevier Science), 267-302.

Kray, J., and Lindenberger, U. (2000). Adult age differences in task switching. Psychol. Aging 15, 126-147.

Liepelt, R., Strobach, T., Frensch, P., and Schubert, T. (2011). Improved intertask coordination skills after extensive dual-task practice. Q. J. Exp. Psychol. 64, 1251-1272.

Lindenberger, U., and Baltes, P. B. (1995). Testing-the-limits and experimental simulation: two methods to explicate the role of learning in development. Hum. Dev. 38, 349-360.

Lindenberger, U., Kliegl, R., and Baltes, P. B. (1992). Professional expertise does not eliminate age differences in imagery-based memory performance during adulthood. Psychol. Aging 7, 585-593.

Logan, G. D., and Gordon, R. D. (2001) Executive control of visual attention in dual-task situations. Psychol. Rev. 108, 393-434.

Maquestiaux, F., Hartley, A. A., and Bertsch, J. (2004). Can practice overcome age-related differences in the psychological refractory period effect? Psychol. Aging 19, 649-667.

Maquestiaux, F., Laguë-Beauvais, M., Ruthruff, E., and Bherer, L. (2008). Bypassing the central bottleneck after single-task practice in the psychological refractory period paradigm: evidence for task automatization and greedy resource recruitment. Mem. Cognit. 36, 1262-1282.

Maquestiaux, F., Laguë-Beauvais, M., Ruthruff, E., Hartley, A. A., and Bherer, L. (2010). Learning to bypass the central bottleneck: declining automaticity with advancing age. Psychol. Aging 25, 177-192.

McDowd, J. M., and Shaw, R. J. (2000). "Attention and aging: a functional perspective," in The Handbook of Aging and Cognition, 2nd Edn, eds F. M. Craik, T. A. Salthouse, F. M. Craik, and T. A. Salthouse (Mahwah, NJ: Lawrence Erlbaum Associates Publishers), 221-292.

Meyer, D. E., and Kieras, D. E. (1999). "Précis to a practical unified theory of cognition and action: some lessons from EPIC computational models of human multiple-task performance," in Attention and Performance XVII: Cognitive Regulation of Performance: Interaction of Theory and Application, eds D. Gopher, A. Koriat, D. Gopher, and A. Koriat (Cambridge, MA: The MIT Press), 17-88.

Nyberg, L., Marklund, P., Persson, J., Cabeza, R., Forkstam, C., Petersson, K., and Ingvar, M. (2003). Common prefrontal activations during working memory, episodic memory, and semantic memory. Neuropsychologia 41, 371-377.

Oldfield, R. C. (1971). The assessment and analysis of handedness: the Edinburgh inventory. Neuropsychologia 9, 102-107.

Pashler, H. (1994). Dual-task interference in simple tasks: data and theory. Psychol. Bull. 116, 220-244.

Raz, N. (2000). "Aging of the brain and its impact on cognitive performance: integration of structural and functional findings," in The Handbook of Aging and Cognition, eds F. I. M. Craik and T. A. Salthouse (Mahwah, NJ: Lawrence Erlbaum Associates), 1-90. 
Riby, L. M., Perfect, T. J., and Stollery, B. T. (2004). The effects of age and task domain on dual task performance: a meta-analysis. Eur. J. Cogn. Psychol. $16,868-891$.

Rogers, R. D., and Monsell, S. (1995). Costs of a predictable switch between simple cognitive tasks. J. Exp. Psychol. Gen. 124, 207-231.

Ruthruff, E., Johnston, J. C., and Van Selst, M. V. (2001). Why practice reduces dual-task interference. $J$. Exp. Psychol. Hum. Percept. Perform. 27, 3-21.

Ruthruff, E., Johnston, J. C., Van Selst, M. V., Whitsell, S., and Remington, R. (2003). Vanishing dualtask interference after practice: has the bottleneck been eliminated or is it merely latent? J. Exp. Psychol. Hum. Percept. Perform. 29, 280-289.

Ruthruff, E., Van Selst, M., Johnston, J. C., and Remington, R. W. (2006). How does practice reduce dual-task interference: integration, automatization, or simply stage-shortening? Psychol. Res. 70, 125-142.

Schubert, T. (1999). Processing differences between simple and choice reaction affect bottleneck localization in overlapping tasks. J. Exp. Psychol. Hum. Percept. Perform. 25, 408-425.
Schubert, T. (2008). The central attentional limitation and executive control. Front. Bisoci. 13, 3569-3580.

Schumacher, E. H., Seymour, T. L., Glass, J. M., Fencsik, D. E., Lauber, E. J., Kieras, D. E., and Meyer, D. E. (2001). Virtually perfect time sharing in dual-task performance: uncorking the central cognitive bottleneck. Psychol. Sci. 12, 101-108.

Sigman, M., and Dehaene, S. (2006). Dynamics of the central bottleneck: dual-task and task uncertainty. PLoS Biol. 4, e220. doi:10.1371/journal.pbio.0040220

Somberg, B. L., and Salthouse, T. A. (1982). Divided attention abilities in young and old adults. J. Exp. Psychol. Hum. Percept. Perform. 8, 651-663.

Strobach, T., Frensch, P. A., and Schubert, T. (2008). "The temporal stability of skilled dual-task performance," in Cognitive Science 2007. Saarbrücken: Proceedings of the 8th Annual Conference of the Cognitive Science Society of Germany, eds H. D. Zimmer, C. Frings, A. Mecklinger, B. Opitz, M. Pospeschill, and D. Wentura.

Strobach, T., Liepelt, R., Schubert, T., and Kiesel, A. (2012a). Task switching: effects of practice on switch and mixing costs. Psychol. Res. 76, 74-83.
Strobach, T., Frensch, P. A., Müller, H., and Schubert, T. (2012b). Age- and practice-related influences on dualtask costs and compensation mechanisms under optimal conditions for dual-task performance. Aging Neuropsychol. Cogn. 19, 222-247.

Strobach, T., Frensch, P. A., Soutschek, A., and Schubert, T. (in press). Investigation on the improvement and transfer of dual-task coordination skills. Psychol. Res.

Tombu, M., and Jolicoeur, P. (2004) Virtually no evidence for virtually perfect time-sharing. J. Exp. Psychol. Hum. Percept. Perform. 30, 795-810.

Van Selst, M., and Jolicoeur, P. (1997). Decision and response in dualtask interference. Cogn. Psychol. 33, 266-307.

Van Selst, M., Ruthruff, E., and Johnston, J. C. (1999). Can practice eliminate the psychological refractory period effect? J. Exp. Psychol. Hum. Percept. Perform. 25, 1268-1283.

Verhaeghen, P. (2011). Aging and executive control: reports of a demise greatly exaggerated. Curr. Dir. Psychol. Sci. 20, 174-180.

Verhaeghen, P., Steitz, D. W., Sliwinski, M. J., and Cerella, J. (2003) Aging and dual-task performance: a meta-analysis. Psychol. Aging 18 , 443-460.
Weiß, R. H. (2006). CFT 20-R: Grundintelligenztest Skala 2 - Revision. Göttingen: Hogrefe.

West, R. L. (1996). An application of prefrontal cortex function theory to cognitive aging. Psychol. Bull. 120, 272-292.

Conflict of Interest Statement: The authors declare that the research was conducted in the absence of any commercial or financial relationships that could be construed as a potential conflict of interest.

Received: 28 November 2011; paper pending published: 05 January 2012; accepted: 18 February 2012; published online: 05 March 2012.

Citation: Strobach T, Frensch P, Müller $H J$ and Schubert $T$ (2012) Testing the limits of optimizing dualtask performance in younger and older adults. Front. Hum. Neurosci. 6:39. doi 10.3389/fnhum.2012.00039

Copyright (c) 2012 Strobach, Frensch, Müller and Schubert. This is an openaccess article distributed under the terms of the Creative Commons Attribution Non Commercial License, which permits non-commercial use, distribution, and reproduction in other forums, provided the original authors and source are credited. 